\title{
INFLUENCE OF THE QUENCHING RATE ON THE STRUCTURE AND MAGNETIC PROPERTIES OF THE Fe-BASED AMORPHOUS ALLOY
}

This paper presents the results of investigations into the structure, microstructure and magnetic properties of $\mathrm{Fe}_{61} \mathrm{Co}_{10} \mathrm{Y}_{8} \mathrm{~W}_{1} \mathrm{~B}_{20}$ amorphous alloy. The alloy samples were in two physical forms: (1) plates of approximate thickness 0.5 $\mathrm{mm}$ (so-called bulk amorphous alloys) and (2) a ribbon of approximate thickness $35 \mu \mathrm{m}$ (so-called classic amorphous alloy). The investigations comprised: X-ray diffractometry, Mössbauer spectrometry, transmission electron microscopy, and selected magnetic measurements; all of the investigations were carried out on samples in the as-quenched state. Analysis of the obtained SEM and TEM images, X-ray diffraction patterns, Mössbauer spectrometry results and measurements of the magnetisation in a high magnetic field facilitated collectively the detailed description of the structure of the investigated alloy, which was found to depend on the quenching speed.

Keywords: Transmissions electron microscopy; Scanning electron microscopy; Amorphous matrials; Free volumes; Quasidislocation

\section{Introduction}

To find the real structure of amorphous alloys is a very complicated task because the iron-based amorphous alloys are usually materials characterised by metastable phases. In this type of alloy, separation of the metastable phases occurs in the so-called supercooled liquid range $\Delta T$ above $55 \mathrm{~K}: \Delta T=$ $T_{x}-T_{g}$, where $T_{x}$ - crystallisation onset temperature $T_{g}$ - glass transition temperature) [1-4]. Based on iron, the ferromagnetic amorphous alloys, exhibiting soft magnetic properties, usually go through primal crystallisation [5-8]. The crystallising phase is rich in iron and placed within the liquid phase subjected to phase separation. The crystallisation mechanism is directly connected with a high value of $\Delta T$. This means that, within their volume, the amorphous alloys have numerous composition fluctuations, created during the super cooling process, below the metastable range of non-mixing.

The value of the parameter $\Delta T$ depends, not only on the chemical composition of the alloy, but also on many other related factors, including the quenching speed of the molten alloy $[9,10]$. The quenching speed of the molten alloy to the solid state determines the structural relaxations occurring in the alloy volume, which, in turn, strongly influences the properties of the resulting amorphous alloys $[11,12]$. An increase in the value of the viscosity of the alloy during the manufacturing process results in the restriction of the magnitude and rate of atomic diffusion. According to the investigations performed in [11-14], the structure and microstructure of samples produced at different cooling speeds influence directly the magnetic properties, despite the fact that the samples possess an amorphous structure. This conclusion proves that the atomic structure of amorphous materials depends on the cooling speed during the production process. By means of the scanning electron microscopy (SEM) study, information about the types of breakthroughs of the amorphous alloys can be obtained. This will allow for comparison of the degree of relaxation of the amorphous structure $[15,16]$. The degree of relaxation of the amorphous structure is very important as it affects the magnetic properties of the alloy [13]. Images from the high resolution transmission electron microscopy (HRTEM) and electron diffraction patterns for the investigated area reveal the structure of the alloy [17]. The other considered methods of investigating the structure of the amorphous alloys are X-ray diffractometry (XRD) and Mössbauer spectrometry [18]. Connecting these four methods facilitates the accurate description of the structure of the amorphous alloys. In the case of iron-based amorphous alloys which exhibit soft magnetic properties, a means for indirect comparison of their structure exists $[19,20]$. Analysis of the saturation magnetisation curves of the amorphous ferromagnetic alloys allows investigation of the effect of structural defects on the magnetisation process while approaching ferromagnetic saturation $[19,20]$. In respect of the amorphous and nanocrystalline alloys, the process of magnetisation, in a strong magnetic field, can be described by the following equation [20]:

$\mu_{0} M(H)=\mu_{0} M_{s}\left[1-\frac{a_{1 / 2}}{\left(\mu_{0} H\right)^{1 / 2}}-\frac{a_{1}}{\left(\mu_{0} H\right)^{1}}-\frac{a_{2}}{\left(\mu_{0} H\right)^{2}}\right]+b\left(\mu_{0} H\right)^{1 / 2}$

where:

$M_{s}-$ spontaneous magnetisation, $\mu_{0}-$ the magnetic permeability of a vacuum, $H$ - magnetic field, $a_{i}(i=1 / 2,1,2)$ - coefficients of the linear fit for the free volumes and linear defects, $b$ - coefficient of the linear fit related with dumping of the

\footnotetext{
* CZESTOCHOWA UNIVERSITY OF TECHNOLOGY, INSTITUTE OF PHYSICS, 19 ARMII KRAJOWEJ AV., 42-200 CZESTOCHOWA, POLAND

\# Corresponding author: nmarcell@wp.pl
} 
spin waves by strong magnetic field, the so-called HolsteinPrimakoff paraprocess [20, 21].

In the ferromagnetic materials, the presence of structural defects in the forms of free volumes and linear defects leads to non-collinear distribution of the magnetic moments (Fig. 1) [20].

These defects are the source of internal stresses and areas of reduced density. That is why complementary investigations utilising all of the aforementioned methods are needed in order to get a complete description of the real amorphous structure.

Therefore, in this paper, the results of the investigations into the classic and bulk $\mathrm{Fe}_{61} \mathrm{Co}_{10} \mathrm{Y}_{8} \mathrm{~W}_{1} \mathrm{~B}_{20}$ amorphous alloy are presented; with coverage of the structural properties (using: SEM, HRTEM, XRD and Mössbauer Spectrometry) and the magnetic properties (using: the Kronmüller theorem of approach to ferromagnetic saturation and the HolsteinPrimakoff paraprocess).

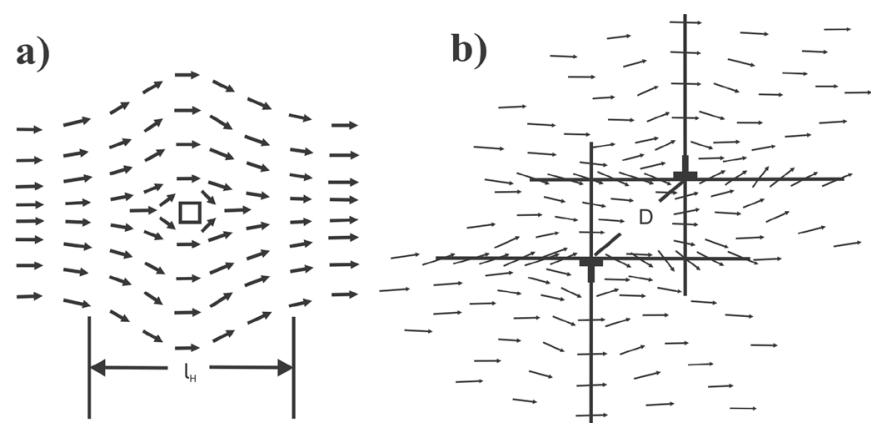

Fig. 1. Distribution of the magnetic moments in the vicinity of (a) the free volumes and (b) quasidislocation dipoles [20][20]

\section{Experimental procedure}

The components used in the production of the amorphous alloys were of high purity: $\mathrm{Fe}-99.99$ at.\%, Co - 99.99 at.\%, Y - 99.99 at.\%, and $\mathrm{W}-99.999$ at.\%. The element, boron, was added in the form of $\mathrm{Fe}_{45.6} \mathrm{~B}_{54.4}$ alloy. Samples used in the investigations were made using two methods: a suctioncasting method (where the molten alloy is sucked into a watercooled, copper die) and the melt-spinning method (involving the unidirectional quenching of the molten alloy on a rotating copper wheel) (Fig. 1). The production process consisted of two steps. In the first stage, under a protective atmosphere of inert gas, the crystalline alloy was prepared by arc-melting the elements. The resulting crystalline alloy was divided into portions (each of which weighed a few grams) and the portions then were used in the second stage of production, in which samples were manufactured in the final forms of ribbons and plates.

The structure of the samples was investigated by means of a BRUCKER ADVANCED D8 X-ray diffractometer, equipped with a $\mathrm{CuK}_{\alpha}$ source. The samples were scanned over the $2 \Theta$ range from 30 to $100^{\circ}$ with a measurement step of $0.02^{\circ}$ and an exposure time of $14 \mathrm{~s}$ per step. The microstructure of the ribbon and plate shaped samples was investigated usinga POLON Mössbauer spectrometer equipped witha ${ }^{57} \mathrm{Co}$ radiation source, the latter being enclosed in the Rh matrix and featuring a halflife of 270 days and an intensity of $50 \mathrm{mCi}$. Both the XRD and Mössbauer investigations were performed on samples which had been powdered using a low-energy process. Using powdered samples allowed information to be obtained from the whole volume of the sample. The microstructure of the samples also was investigated by means of SEM and HRTEM. Images of the breakthroughs were obtained using the maximal resolution of 25,000x, using a Supra 25 Zeiss scanning electron microscope, the latter utilising detection of the secondary electrons with an acceleration voltage of $25 \mathrm{kV}$. Images of the samples, the latter being in the form of lamels, were obtained using a high resolution electron microscope FEI S/TEM TITAN 80-300 with an acceleration voltage of $330 \mathrm{kV}$. The lamels used in the investigations were prepared for scanning by etching using a gallium ion beam with an energy level of $5 \mathrm{keV}$. The magnetic investigations were performed by a LakeShore 7003 vibrating sample magnetometer (VSM) using a magnetic field of up to $2 \mathrm{~T}$. Analysis of the initial magnetisation curves was performed according to the $\mathrm{H}$. Kronmüller theorem.

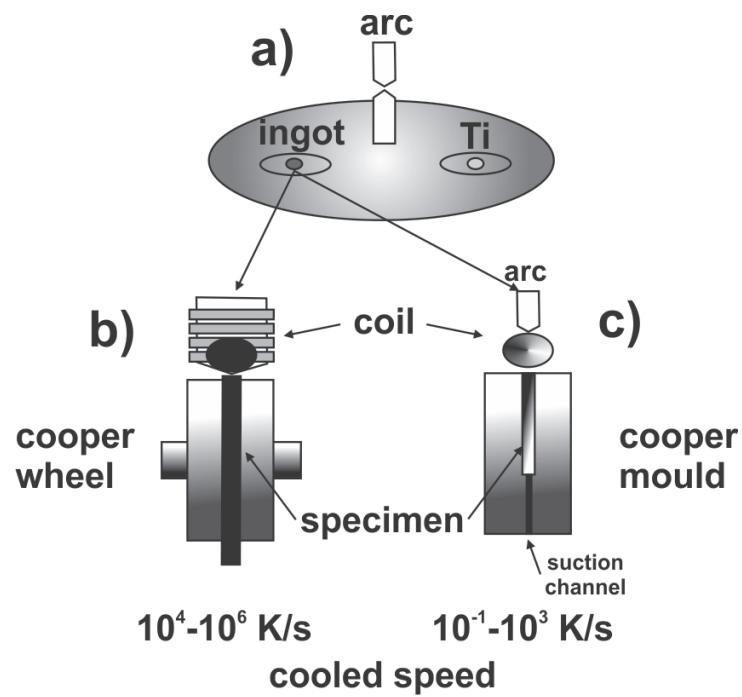

Fig. 2. A schematic description of the production cycle for conventional and bulk amorphous materials: (a) the initial preparation of polycrystalline ingots using arc-melting; (b) the amorphous stripcasting process, (c) the process of production for creation of bulk tiles using the suction-casting method

\section{Results and discussion}

In Fig. 3, X-ray diffraction patterns are presented for the samples made from the plate and ribbon of the investigated alloy, $\mathrm{Fe}_{61} \mathrm{Co}_{10} \mathrm{Y}_{8} \mathrm{~W}_{1} \mathrm{~B}_{20}$.

The diffraction patterns, presented in Fig. 3, are similar and each exhibits a single, broad diffraction maximum, occurring at the $2 \Theta$ point from $\sim 35-50^{\circ}$. This is typical of the iron-based amorphous alloys. The transmission Mössbauer spectra, and corresponding hyperfine field distributions, are presented in Fig. 4.

The Mössbauer spectra obtained for the samples of $\mathrm{Fe}_{61} \mathrm{Co}_{10} \mathrm{Y}_{8} \mathrm{~W}_{1} \mathrm{~B}_{20}$ alloy are typical of ferromagnetic materials with an amorphous structure. Each spectrum consists of six wide and asymmetrical Zeeman's lines. Obtained from analysis of the Mössbauer spectra, the hyperfine field distributions on the ${ }^{57} \mathrm{Fe}$ nuclei consist of clearly separated low- and high - field components. This analysis has been performed taking into account the distribution of the hyperfine field which, in turn, describes the local atomic distribution of the Fe atoms. The 
data obtained from analysis of the transmission Mössbauer spectra are presented in Table 1.



Fig. 3. X-ray diffraction patterns for the investigated alloy: (a) ribbon, (b) plate
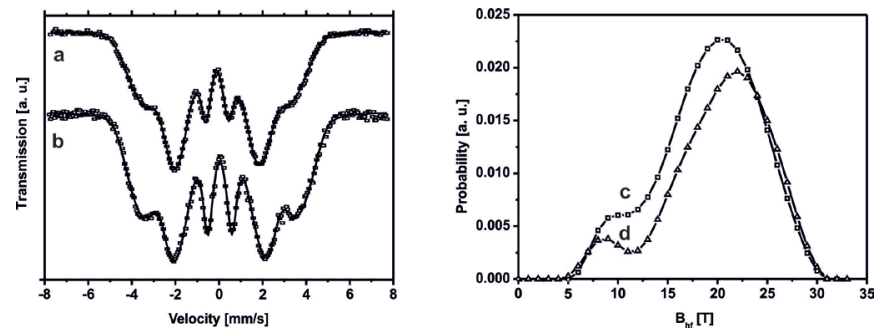

Fig. 4. (a, b) The transmission Mössbauer spectra and (c,d) corresponding hyperfine field distributions on the 57Fe nuclei for the investigated alloy: $(a, c)$ ribbon; $(b, d)$ plate

TABLE 1

The value of the mean hyperfine field using the ${ }^{57} \mathrm{Fe}$ nuclei $\left(B_{e f}\right)$ and dispersion of the hyperfine field distributions of the amorphous phase $\left(D_{a m}\right)$.

\begin{tabular}{|c|c|c|c|}
\hline Form & $B_{e f}(\mathrm{~T})$ & $D_{a m}(\mathrm{~T})$ & Ref. \\
\hline Ribbon & $19.19(2)$ & $5.001(3)$ & {$[22]$} \\
\hline Plate (suction-casting) & $20.19(2)$ & $5.059(3)$ & {$[22]$} \\
\hline
\end{tabular}

The higher value of the average induction of the hyperfine field for the plate-shaped sample suggests a higher value of atomic packing density, in comparison with the ribbon-shaped sample. In turn, this suggests a higher degree of order in the topological structure of the plate-shaped sample. The observed differences, between the two samples, are the result of the different cooling rates of the molten alloy during the production process. A longer quenching time supports relaxation processes, which are related with the atomic diffusion. During the quenching process, the diffusion of atoms within the metallic alloy leads to temporary or full stabilisation of the structure and also to division of the phases, which are characterised by non-mixing in the metastable state. The dispersion of the hyperfine field distributions for the plateshaped sample is higher than that for the ribbon-shaped sample; this suggests a lower value of homogeneity in the vicinity of the iron atoms. The distribution of the hyperfine field on the 57Fe nuclei covers the same range for both samples, but differs slightly in shape. For both samples, the low- and high-field components can be distinguished. The maximum of the highfield component clearly depends on the production method and is higher for the sample obtained at the lower cooling speed. The low-field components have maximal values at the induction of the hyperfine field range from $6-8 \mathrm{~T}$. The separated low-field components are related to the presence of the yttrium atoms in the nearest neighbourhood of the iron atoms. Yttrium atoms are located in the first co-ordination zone and are responsible for lowering the value of the hyperfine field [18, 23]. SEM images, showing the surfaces of the breakthroughs in the ribbon- and plate-shaped samples of the investigated $\mathrm{Fe}_{61} \mathrm{Co}_{10} \mathrm{Y}_{8} \mathrm{~W}_{1} \mathrm{~B}_{20}$ alloy, are presented in Fig. 5 and Fig. 6. Fig. 5 shows the overall view of the breakthrough of the ribbon-shaped sample. In the indicated area, a smooth breakthrough can be seen; this type dominates on the ribbon cross-section (Fig. 5b). A completely opposite character of breakthrough can be observed for the plate-shaped sample. Fig. 6 shows the general view of the breakthrough, with areas indicated for further observations. A place with missing material volume, created during the production process, can be distinguished (the area indicated within the black circle). In the vicinity of this imperfection, only a smooth breakthrough has been observed. Closer to the sample edge, where the copper die had been fully filled by the alloy (two black squares), a mixed breakthrough has been created during the decohesion process. In Fig. 6c, the dominant type of breakthrough is smooth; however, the clear scale-like, well-developed breakdown with a fine vein-like surrounding structure can be observed. This type of breakthrough can suggest various degrees of relaxation within the structure. Fig. 6 and d shows the structure of the breakthrough very near the sample edge. In Fig. 6b, the line creating the division band of the breakthrough is clearly visible with a well-developed scale-like structure along it. Within these scale-like structures the breakthrough has been more complicated and it could be described as a stream-like breakthrough. The presented images of the breakthroughs in the investigated samples suggest that, in the amorphous materials, the quality of the structure and its degree of relaxation depend on the production process and more precisely - on the quenching speed of the molten alloy.

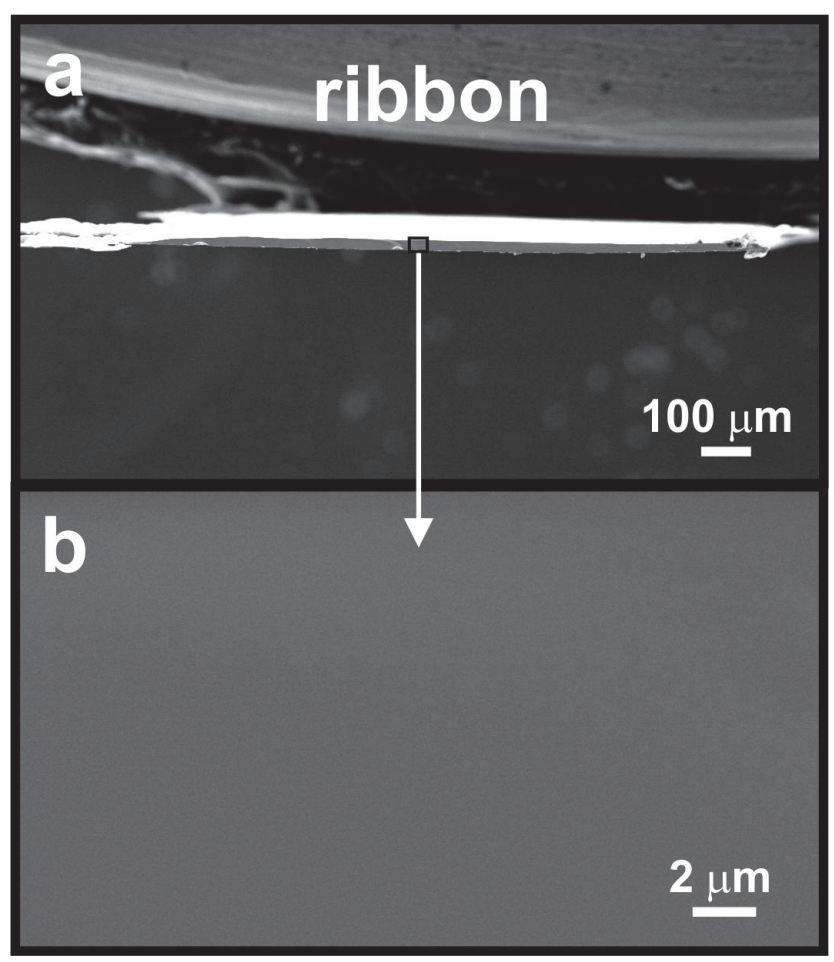

Fig. 5. (a) The SEM image of the ribbon-shaped sample breakthrough and (b) magnification of the indicated area. 


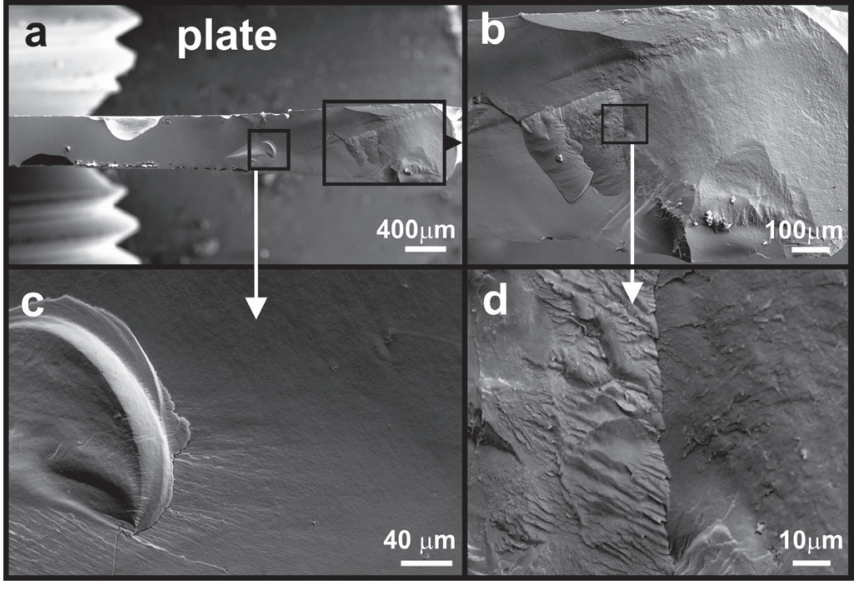

Fig. 6. (a) The SEM image of the plate-shaped sample breakthrough and ( $b, c$ and $d)$ the magnifications of the indicated areas

The amorphicity of the obtained samples of $\mathrm{Fe}_{61} \mathrm{Co}_{10} \mathrm{Y}_{8} \mathrm{~W}_{1} \mathrm{~B}_{20}$ alloy also was confirmed by the transmission electron microscopy investigations (Fig. 7). In the case of the two analysed samples, the obtained area images are similar. In the presented images of the microstructure (Fig. 7 a and c) areas with symmetrical atomic ordering are not visible, which indicates the amorphicity of the samples. The electron diffraction patterns, obtained for selected areas of the samples, provide additional confirmation of theamorphicity of the investigated samples. These patterns are in the form of wide, diffused rings, which is typical for materials with short-range ordering.
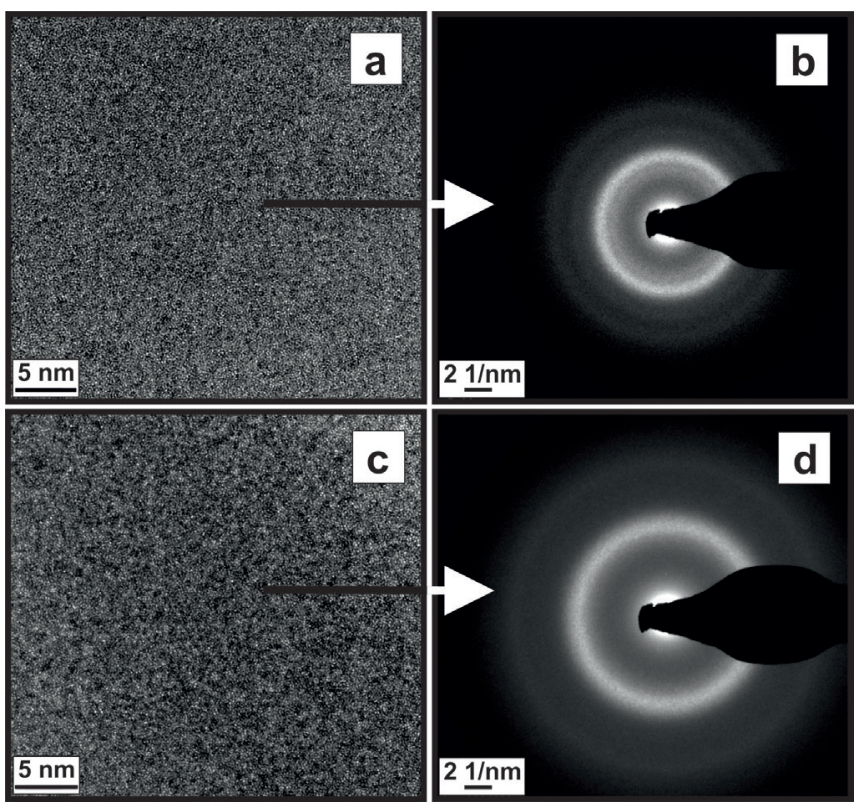

Fig. 7. $(a, c)$ images of the material structures obtained by TEM for the investigated alloy, and (c, d) corresponding electron diffraction patterns STEAM, $(a, b)$ ribbon; $(c, d)$ plate

Utilizing measurements of the magnetisation of the amorphous materials in strong magnetic fields, above the effective anisotropy field in the so-called 'approach to ferromagnetic saturation' area, the effect of the structural defects on the magnetisation process can be established [13, 19, 20]. In Fig. 8, static hysteresis loops are presented for the ribbon and plate-shaped samples of the investigated alloy. The shapes of the static hysteresis loops are similar and typical for ferromagnetic materials with soft magnetic properties. The ribbon-shaped sample is characterised by substantially higher values of initial magnetic susceptibility and saturation magnetization, and a lower value of coercive field. Again, it can be seen that the quenching speed, during the production process, has a significant impact on the microstructure and magnetic properties of the investigated alloy. Data obtained from analysis of the static hysteresis loops are presented in Table 2 .

TABLE 2

Data obtained from analysis of the static hysteresis loops: saturation magnetization $-\mu_{0} M_{s}$, coercive field $-H_{c}$

\begin{tabular}{|c|c|c|c|}
\hline \hline Form & $\mu_{0} M_{s}(\mathrm{~T})$ & $H_{c}(\mathrm{~A} / \mathrm{m})$ & Ref. \\
\hline Ribbon & $1.49(2)$ & $61(3)$ & {$[12]$} \\
\hline Plate & $1.14(2)$ & $37(3)$ & {$[22]$} \\
\hline
\end{tabular}

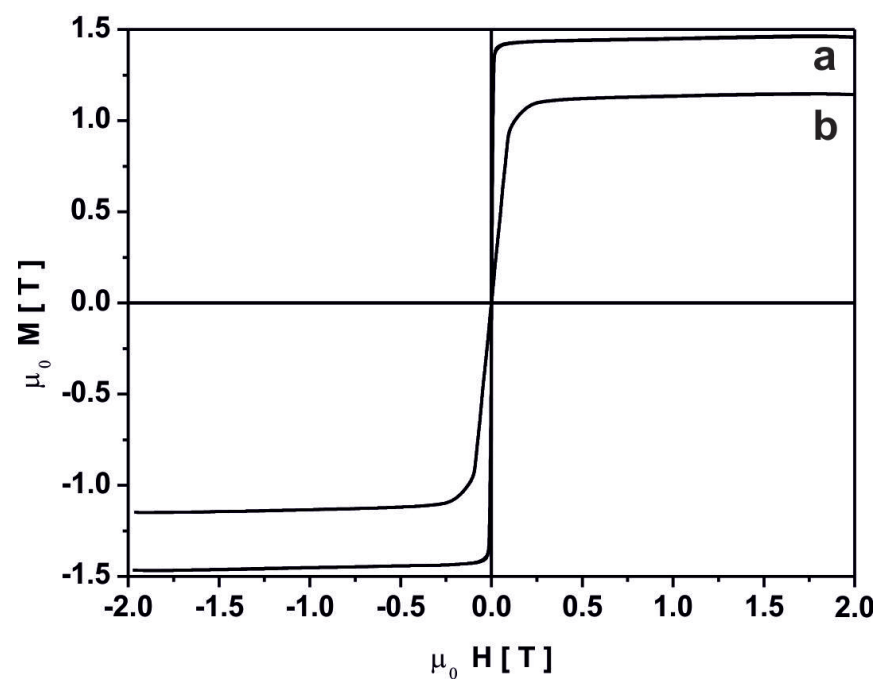

Fig. 8.The static magnetic hysteresis loops, recorded for the samples of the investigated alloy: (a) ribbon, (b) plate

The initial magnetisation curves, high-field magnetisation curves as functions of $(\mu 0 \mathrm{H})^{-1}$ and $(\mu 0 \mathrm{H})^{-2}$, and linear fit of the magnetisation as a function of $(\mu \mathrm{H})^{1 / 2}$, describing the HolsteinPrimakoff paraprocess, are presented in Fig. 9. For the ribbonshaped sample, a good linear fit has been obtained for the second law of the approach to ferromagnetic saturation within the field range of $0.01 \mathrm{~T}$ to $0.13 \mathrm{~T}$. In the case of the plate-shaped sample, the third law of the approach to ferromagnetic saturation has been fitted over a wide magnetic field range $(0.13 \mathrm{~T}-0.40 \mathrm{~T})$. Under the influence of higher magnetic fields, $0.13 \mathrm{~T}$ for the ribbon sample and $0.40 \mathrm{~T}$ for the plate sample, the HolsteinPrimakoff paraprocess, related with the dumping of thermally induced spin waves, has been observed. 

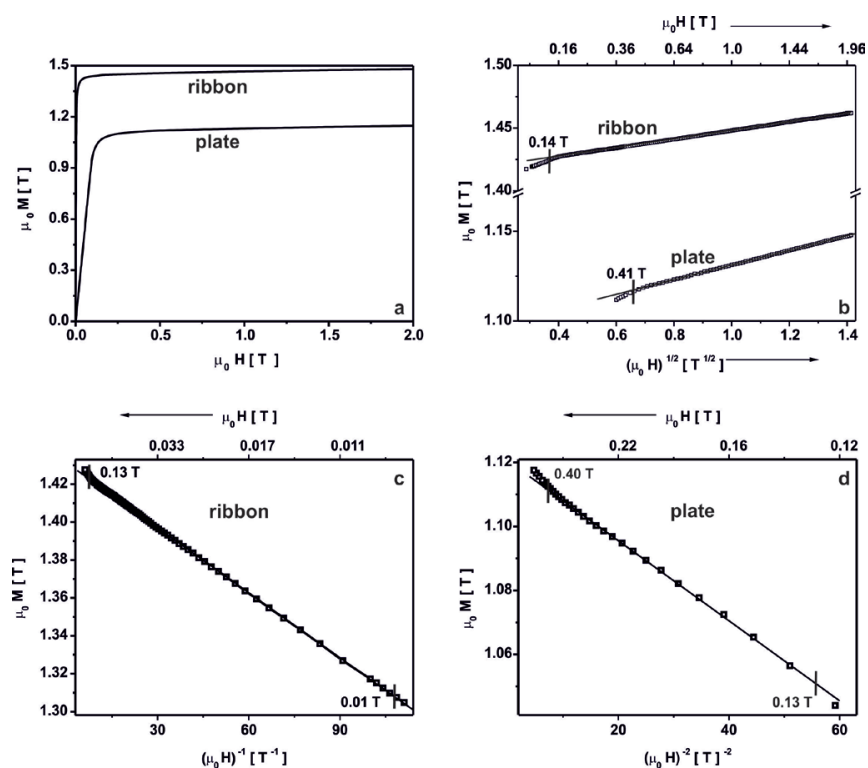

Fig. 9. (a) The initial magnetisation curves, (b) HolsteinPrimakoff paraprocess, and high-field magnetisation as a function of the magnetizing field in the powers of: (c) $(\mu 0 \mathrm{H})$-1and (d) $(\mu 0 \mathrm{H})-2$; for the investigated alloy in the as-quenched state in the forms of a ribbon and a plate

Data obtained from analysis of the initial magnetisation curves, according to the $\mathrm{H}$. Kronmüller theorem, are presented in Table 3.

According to Kaul [24] and Corb [25], in amorphous alloys featuring a highly relaxed structure there are constant changes occurring in the vicinity of the central magnetic atom. In such a relaxed structure each magnetic atom has $\approx 12$ neighbours, and in a structure which is not relaxed the equivalent value is only 9-10 neighbours. An increase in the value of the parameter describing the stiffness of the spin wave is associated with an increase in the number of atoms in the nearest neighbourhood of the central magnetic atom. According to the data gathered in Table 3, the sample made by unidirectional quenching of the molten alloy on a copper wheel possesses the more homogeneous magnetic structure.

\section{Conclusions}

In this paper, the structure, microstructure and magnetic properties of $\mathrm{Fe}_{61} \mathrm{Co}_{10} \mathrm{Y}_{8} \mathrm{~W}_{1} \mathrm{~B}_{20}$ amorphous alloy have been studied. The samples were made in two forms: a ribbon of approximate thickness $30 \mu \mathrm{m}$ and a plate of approximate thickness $0.5 \mathrm{~mm}$. It has been confirmed that the manufactured samples, in the as-quenched state, were fully amorphous. The amorphous ribbons were made using a cooling speed of $10^{4}-10^{6} \mathrm{~K} / \mathrm{s}$, whereas the bulk amorphous sample in plate- form was cooled at $10^{1}-10^{4} \mathrm{~K} / \mathrm{s}$. On the basis of the performed investigations, it could be stated that the cooling speed employed during the production process plays a major role in the formation of the structural and magnetic properties of the iron-based alloys. The Mössbauer investigations facilitate the collection of information about the nearest neighbourhood of the Fe atoms. Changes in the spacial configuration of the elements, caused by redistribution of the magnetic moments of the atomic pairs (Fe-Fe, Fe-Co, $\mathrm{Co}-\mathrm{Co}$ ) and surrounding them pairs of $(\mathrm{Fe}-\mathrm{Y})$ exclusively result from the production method. The SEM images of the breakthroughs for the ribbonshaped and plate-shaped samples differ, and this fact is also related with the sample manufacturing process. Restricting the quenching time of the molten alloy limits the processes of atomic diffusion, which additionally are hindered by an increase in the viscosity value of the solidifying alloy with high glass-forming ability. Analysis of the breakthroughs for both alloy samples revealed that, for each sample, the structural relaxation process proceeds in a different way and with different strength. The images of the microstructure of selected areas of the samples, captured using TEM, and the corresponding electron diffraction patterns, are typical for the amorphous materials. On the basis of the H. Kronmüller theorem, the influence of the structural defects on the magnetic properties has been evaluated. Also, the spin wave stiffness parameter has been calculated; this gives an indication of the nearest neighbourhood of the central $\mathrm{Fe}$ atom and the shortrange ordering. It has been found that the value of $D_{s p}$ for the ribbon-shaped sample is much higher, which means that the magnetic structure of this sample is more ordered.

During the production process of an amorphous alloy, the quenching rate is a factor which is instrumental in deciding the microstructure and magnetic properties of the resulting alloy.

\section{REFERENCES}

[1] X.M. Huang, C.T. Chang, Z.Y. Chang, X.D. Wang, Q.P. Cao, B.L. Shen, A. Inoue, J.Z. Jiang, Formation of bulk metallic glasses in the $\mathrm{Fe}-\mathrm{M}-\mathrm{Y}-\mathrm{B}(\mathrm{M}=$ transition metal $)$ system, $\mathrm{J}$. Alloys. Compd. 460, 708-713 (2008).

[2] Haitao Miao, Chuntao Chang, Yanhui Li, Yingmin Wang, Xingjie Jia, Wei Zhang, Fabrication and properties of soft magnetic $\mathrm{Fe}-\mathrm{Co}-\mathrm{Ni}-\mathrm{P}-\mathrm{C}-\mathrm{B}$ bulk metallic glasses with high glass-forming ability, J. Non-Cryst. Solids 421, 24-29 (2015).

[3] H.S. Chen, Thermodynamic considerations on the formation and stability of metallic glasses, Acta Metall. 22, 1505-1511 (1974).

[4] H.B. Wang, L.X. Ma, L. Li, B. Zhang, Fabrication of Fe-based bulk metallic glasses from low-purity industrial raw materials,

TABLE 3

Data obtained from analysis of magnetization as a function of magnetic field to the powers of: $-1,-2$ and $1 / 2 . D_{s p}-$ spin-wave stiffness parameter, $N_{\text {dip }}$ - density of quasidislocational dipoles

\begin{tabular}{|c|c|c|c|c|c|c|}
\hline \hline Form & $\begin{array}{c}a_{1} \\
\left(10^{-2} \mathrm{~T}^{1}\right)\end{array}$ & $\begin{array}{c}a_{2} \\
\left(10^{-2} \mathrm{~T}^{2}\right)\end{array}$ & $\begin{array}{c}b \\
\left(10^{-2} \mathrm{~T}^{1 / 2}\right)\end{array}$ & $\begin{array}{c}D_{s p} \\
\left(10^{-2} \mathrm{meV} \mathrm{nm}^{2}\right)\end{array}$ & $\begin{array}{c}N_{\text {dip }} \\
\left(10^{16} \mathrm{~m}^{-2}\right)\end{array}$ & $\begin{array}{c}\text { Ref. } \\
\text { Ref }\end{array}$ \\
\hline Ribbon & $0.104(7)$ & - & $3.43(1)$ & $62.92(3)$ & $56.23(2)$ & - \\
\hline Plate & - & $0.11(2)$ & $4.06(1)$ & $22]$ & {$[22]$} \\
\hline
\end{tabular}


J.Alloys. Compd. 62，1-4 (2015).

[5] K. Błoch, Magnetic properties of the suction-cast bulk amorphous alloy: $\left(\mathrm{Fe}_{0.61} \mathrm{Co}_{0.10} \mathrm{Zr}_{0.025} \mathrm{Hf}_{0.025} \mathrm{Ti}_{0.02} \mathrm{~W}_{0.02} \mathrm{~B}_{0.20}\right)_{96} \mathrm{Y}_{4}, \mathrm{~J}$. Magn. Magn. Mater. 390, 118-122 (2015).

[6] Xingjie Jia, Yanhui Li, Hao Wang, Guoqiang Xie, Shinichi Yamaura, Wei Zhang, Synthesis and properties of ferromagnetic Fe-based (Fe, Ni, Co)- Mo- P- C- B bulk metallic glasses with large supercooled liquid region, Physica B, http://dx.doi. org/10.1016/j.physb.2015.05.004

[7] X.H. Tan, H. Xu, Q. Bai, W.J. Zhao, Y.D. Dong, Magnetic properties of $\mathrm{Fe}-\mathrm{Co}-\mathrm{Nd}-\mathrm{Y}-\mathrm{B}$ magnet prepared by suction casting, J. Non-Cryst. Solids 353, 410-412 (2007).

[8] Q.J. Chen, H.B. Fan, L. Ye, S. Ringer, J.F. Sun, J. Shen, D.G. McCartney, Enhanced glass forming ability of $\mathrm{Fe}-\mathrm{Co}-$ Zr-Mo-W-B alloys with Ni addition, Mat. Sci. Eng. A 402, 188-192 (2005).

[9] X.M. Huang, X.D. Wang, Y. He, Q.P. Cao and J.Z. Jiang, Are there two glass transitions in $\mathrm{Fe}-\mathrm{M}-\mathrm{Y}-\mathrm{B}(\mathrm{M}=\mathrm{Mo}, \mathrm{W}, \mathrm{Nb})$ bulk metallic glasses? Scripta Mater. 60 , 152-155 (2009).

[10] A. Inoue, High strength bulk amorphous alloys with low critical cooling rates, Materi.Trans., JIM 36, 866-875 (1995).

[11] M. Nabialek, P. Pietrusiewicz, M.J. Dospial, M. Szota, K. Błoch, K. Gruszka, K. Oźga, S. Garus, Effect of manufacturing method on the magnetic properties and formation of structural defects in $\mathrm{Fe}_{61} \mathrm{Co}_{10} \mathrm{Y}_{8} \mathrm{Zr}_{1} \mathrm{~B}_{20}$ amorphous alloy, J. Alloy. Compd. 615, 51-55 (2014).

[12] M. Nabiałek, P. Pietrusiewicz, M. Dośpiał, M. Szota, J. Gondro, K. Gruszka, A. Dobrzańska-Danikiewicz, S. Walters, A. Bukowska, Influence of the cooling speed on the soft magnetic and mechanical properties of $\mathrm{Fe}_{61} \mathrm{Co}_{10} \mathrm{Y}_{8} \mathrm{Zr}_{1} \mathrm{~B}_{20}$ amorphous alloy, J. Alloy. Compd. 615, 56-60 (2014).

[13] M. Nabiałek, M. Szota, M. Dośpiał, P. Pietrusiewicz, S. Walters, Influence of structural defects on the magnetization process in high-magnetic fields in the $\mathrm{Fe}_{61} \mathrm{Co}_{10} \mathrm{Y}_{8} \mathrm{Nb} 1 \mathrm{~B} 20$ alloy in the form of ribbons and plates, J. Magn. Magn. Mater. 322 (21), 3377-3380, (2010).

[14] Wolfgang Wallisch, Josef Fidler, Peter Toson, Herbert Sassik, Robert Svagera, Johannes Bernardi, Synthesis and characterisation of $(\mathrm{Fe}, \mathrm{Co}) 2-3 \mathrm{~B}$ microcrystalline alloys, J. Alloy. Compd. 644, 199-204(2015).

[15] M. Nabiałek, P. Pietrusiewicz, M. Szota, A. DobrzańskaDanikiewicz, S. Lesz, M. Dośpiał, K. Błoch, K. Oźga, The structural stability of the $\mathrm{Fe}_{36} \mathrm{Co}_{36} \mathrm{Si}_{19} \mathrm{~B}_{5} \mathrm{Nb}_{4}$ bulk amorphous alloy, Arch. Metall. Mater. 59 (1), 259-262 (2014).

[16] Weiming Yang, Haishun Liu, Yucheng Zhao, Akihisa Inoue, Kemin Jiang, Juntao Huo, Haibo Ling, Qiang Li, Baolong Shen, Mechanical properties and structural features of novel Fe-based bulk metallic glasses with unprecedented plasticity, Scientific Reports 4, 6233 (2014)1-6, DOI: 10.1038/srep06233

[17] G.H. Cao, N. Liu, J.C. Peng , X. Li, G.J. Shen, A.M. Russell, Transmission electron microscopy study of the microstructure of a Ti-Fe-Zr alloy, Mater. Charact. 83, 43-48 (2013).

[18] P. Gupta, A. Gupta, A. Shukla, Tapas Ganguli, A. K. Sinha, G. Principi, A. Maddalena, Structural evolution and the kinetics of $\mathrm{Cu}$ clustering in the amorphous phase of $\mathrm{Fe}-\mathrm{Cu}-\mathrm{Nb}-\mathrm{Si}-\mathrm{B}$ alloy, J. Appl. Phys. 110, 033537 (2011).

[19] H. Kronmüller, Micromagnetism and microstructure of amorphous alloy, J. Appl. Phys. 52 (3), 1859-1864 (1981).

[20] H. Kronmüller, M. Fähnle, Micromagnetism and the microstructure of ferromagnetic solids, Cambridge University Press (2003).

[21] T. Holstein, H. Primakoff, Field Dependence of the Intrinsic Domain Magnetization of a Ferromagnet, Phys. Rev. 58, 1098-1113 (1940).

[22] M. Nabiałek, P. Pietrusiewicz, K. Błoch, Influence of the production method of $\mathrm{Fe}_{61} \mathrm{Co}_{10} \mathrm{Y}_{8} \mathrm{Zr}_{1} \mathrm{~B}_{20}$ amorphous alloy on the resulting microstructure and hyperfine field distribution, $\mathrm{J}$. Alloy. Compd. 628, 424-428 (2015).

[23] Y. Hirotsu, T. Hanada, T. Ohkubo, A. Makino, Y. Yoshizawa, T.G. Nieh, Intermetallics 12, 1081-1088 (2004).

[24] N. Kaul, Magnetic properties of amorphous (fe, ni) $80 \mathrm{~B}$ 20,(fe, ni) 80 B 19 si 1, and (fe, ni) 80 P 14 B 6 alloys, IEEE Trans. Magn. 17, 1208-1215 (1981).

[25] B. W. Corb, R. C. O'Handley, N. J. Grant, Chemical bonding, magnetic moments, and local symmetry in transition-metalmetalloid alloys, Phys. Rev. B 27, 636-641 (1983). 